\title{
Anti Aristotle-The Division of Zero by Zero
}

\author{
Jan Pavo Barukčić1, Ilija Barukčić2 \\ ${ }^{1}$ Department of Mathematics and Computer Sciences, University of Münster, Münster, Germany \\ ${ }^{2}$ Internist, Horandstrasse, Jever, Germany \\ Email:j_baru01@uni-muenster.de,Barukcic@t-online.de
}

Received 28 March 2016; accepted 24 April 2016; published 27 April 2016

Copyright (C) 2016 by authors and Scientific Research Publishing Inc.

This work is licensed under the Creative Commons Attribution International License (CC BY).

http://creativecommons.org/licenses/by/4.0/

(c) (i) Open Access

\begin{abstract}
Today, the division of zero by zero $(0 / 0)$ is a concept in philosophy, mathematics and physics without a definite solution. On this view, we are left with an inadequate and unsatisfactory situation that we are not allowed to divide zero by zero while the need to divide zero by zero (i.e. divide a tensor component which is equal to zero by another tensor component which is equal to zero) is great. A solution of the philosophically, logically, mathematically and physically far reaching problem of the division of zero by zero $(0 / 0)$ is still not in sight. The aim of this contribution is to solve the problem of the division of zero by zero $(0 / 0)$ while relying on Einstein's theory of special relativity. In last consequence, Einstein's theory of special relativity demands the division of zero by zero. Due to Einstein's theory of special relativity, it is $(0 / 0)=1$. As we will see, either we must accept the division of zero by zero as possible and defined, or we must abandon Einstein's theory of special relativity as refuted.
\end{abstract}

\section{Keywords}

Number Theory, Quantum Theory, Relativity Theory, Unified Field Theory, Causality

\section{Introduction}

The development of mathematical science is full of contradictions and serious misrepresentations, especially concerning the division of zero (denoted by the sign 0 ) by zero. In contemporary mathematics a division of zero by zero $(0 / 0)$ is called an indeterminate form and still, it is customary to claim that a division of zero by zero $(0 / 0)$ has no defined value. Historically, some kinds of symbols for zero or empty places corresponding in this respect to our zero in the positional representation of numbers were already used by the Babylonians, the Greeks, and the Mayas too. Nevertheless, in many reference works in mathematics, the arithmetic of zero is credited entirely to the Hindu contribution and especially to Brahmagupta.

"The arithmetic of zero is entirely the Hindu contribution to the development of mathematical science, with 
no other early nations do we find any treatment of zero" [1].

In contrast to the statement above, especially Aristotle (384 BC-322 BC), a pupil of Plato, contributed some very important positions concerning the numerical notion of zero and to the result of division by zero. Moreover, Aristotle himself explicitly stated the impossibility of the division by zero just about fifteen hundred years before the time of Bhaskara. A significant passage in Aristotle's Physics is related to the numerical notion of zero and the result of the division by zero too. In Physica, Aristotle wrote:

"Now there is no ratio in which the void is exceeded by body, as there is no ratio of 0 to a number. For if 4 exceeds 3 by 1 , and 2 by more than 1 , and 1 by still more than it exceeds 2 , still there is no ratio by which it exceeds 0 ; for that which exceeds must be divisible into the excess + that which is exceeded, so that 4 will be what it exceeds 0 by +0 . For this reason, too, a line does not exceed a point-unless it is composed of points" [2].

Clearly, in this quotation Aristotle did not look upon zero as a number in the strict sense of the word but had "the arithmetical zero in mind" [3]. Aristotle excluded the division by zero by using the traditional meanings of words. According to Aristotle, if a division by zero was possible, then the result would exceed every possible integer.

To proceed further, some arithmetic operations with zero are allowed. Nicomachus ( $\sim 60-\sim 120$ AD), born in Gerasa (the ancient Roman province of Syria) was influenced by Aristotle's work. In his publication introduction to Arithmetic, Nicomachus [4] claimed that the sum of nothing added to nothing was nothing. According to Nicomachus, be sure that

$$
0+0=0
$$

However, until the sixteenth and seventeenth centuries, zero as such was not fully accepted in algebra. Nevertheless, the earliest, but quite inadequate, consideration and extant reference to the division by zero is ascribed to Brahmagupta (597-668 AD), an Indian mathematician and astronomer. As a matter of fact, Brahmagupta's earliest recorded Indian (Hindu) contributions to explain the division by zero is due to his writing of the Brahmasphutasiddhanta in $628 \mathrm{AD}$ Brahmagupta wrote:

"Positive, divided by positive, or negative by negative, is affirmative. Cipher, divided by cipher, is nought. Positive, divided by negative, is negative. Negative, divided by affirmative, is negative. Positive, or negative, divided by cipher, is a fraction with that for denominator: or cipher divided by negative or affirmative" [5].

It is well known that the Brahmasphutasiddhanta of Brahmagupta leads to some algebraic absurdities. Consequently, around 200 years after Brahmagupta, Mahavira (Mysore, India) tried to revise the Brahmasphutasiddhanta of Brahmagupta. Bhaskara (over 500 years after Brahmagupta) worked on the division by zero too. In contrast to Aristotle's claim of the impossibility of division by zero, the division by zero is given by Bhaskara in 1152 as follows:

"Statement: Dividend 3. Divisor 0. Quotient the fraction 3/0. This fraction, of which the denominator is cipher, is termed an infinite quantity" [6].

In particular, Bhaskara himself did not assert the impossibility of the division by zero. The work of the Hindu mathematicians spread west to the Arabic mathematicians as well as east to China and later to Europe too. In 1247, the Chinese mathematician Qin Jiu-shao (also known as Ch'in Chiu-Shao) introduced the symbol O for zero in his mathematical text 'Mathematical treatise in nine sections'. The number zero is related to infinity. The contemporary viewpoint of infinity is associated with the name John Wallis. In 1655, John Wallis (1616-1703), an English mathematician, introduced the symbol $\infty$ for infinity. According to John Wallis, "estoenim $\infty$ nota numeri infiniti" [7]. Translated into English: "let the symbol $\infty$ denote infinity". In particular, Wallis himself claimed in 1656 " $1 / \infty \ldots$ habendaerit pro nihilo" [8] or

$$
\frac{+1}{+\infty}=+0
$$

Thus far, Wallis [8] is demanding, without a mathematical proof, that

$$
\frac{+1}{+\infty} \times+\infty=+1
$$

Isaac Newton supported the position of Wallis [9] in his book Opuscla. Due to Isaac Newton it is " $1 / 0=$ Infinitae" [10]. George Berkeley (1685-1753), Bishop of Cloyne, claimed that reality (no longer objective) consists exclusively of minds. Another recorded reference to the mathematical impossibility of assigning a value to a di- 
vision by 0 is credited to George Berkeley's criticism of infinitesimal calculus in The Analyst.

"They are neither finite Quantities, nor Quantities infinitely small, nor yet nothing. May we not call them the Ghofts of departed Quantities?" [11].

Berkeley's Analyst was a direct attack on the foundations and principles of the infinitesimal calculus as developed by Newton and Leibniz. Finally, a rigorous foundation for the principles of the infinitesimal calculus was given through the work of the prolific mathematician, Augustin-Louis Cauchy. Cauchy formalized the concept of a limit and created the specialism now called analysis.

Many great mathematicians tried to put an end to the debate concerning the division of zero by zero. But still, we are no [12] closer to finding a solution. Today, the division of zero by zero is a concept in mathematics without [13] a definitive answer. We may ask ourselves, can Einstein's theory of special relativity bring us to the point of admitting or disabling the division of zero by zero, definitely?

\section{Material and Methods}

\subsection{Definitions}

\subsubsection{Definition. Thought Experiments}

The general acceptance, importance and enormous influence of properly constructed (real or) thought experiments (as devices of scientific investigation) is backgrounded by many common features. Especially, the possibility to investigate some basic properties of the nature even under conditions when it is difficult or too expensive to run a real experiment is worth being mentioned. Furthermore, a thought experiment can draw out a contradiction in a theory and thereby refuting the same. Again, it is necessary to highlight the possibility of a thought experiments to provide evidence against or in favor of a theory. However, thought experiments used for diverse reasons in a variety of areas are at the end no substitute for a real experiment. Thus far, real or thought experiments can help us to solve the problem of the division of zero by zero.

\subsubsection{Definition. Proof by Contradiction (Reductio ad Absurdum)}

The logical background of a proof by contradiction is Aristotle's law of non-contradiction. A rigorous proof by contradiction of a theorem follows the standard method of contradiction used in science and mathematics and should be convincing as much as possible. For the first, we assume that a claim/a theorem/a proposition/a statement et cetera which has to be proved, is true. One then proceeds to demonstrate that a conclusion drawn from such a claim/a theorem/a proposition/a statement et cetera leads to a contradiction. Hence, the supposed claim/ theorem/proposition/statement et cetera is deemed to be false. Consequently, we are then led to conclude that it was wrong to assume the claim/the theorem/the proposition/the statement was true. Thus far, the claim/the theorem/the proposition/the statement are proved to be false. Reductio ad absurdum is a widely used technique to expose a fallacy. The logical form of reduction and absurdum is the following:

Assume $\mathrm{P}$ (i.e. an equation, a theorem et cetera) is true.

From this assumption, deduce that $\mathrm{Q}$ (i.e. an equation, a theorem et cetera) is true.

Now deduce somehow that $\mathrm{Q}$ is false.

Thus, P implies both Q and not Q (a contradiction, which is necessarily false).

Therefore, $P$ itself must be false.

\subsubsection{Definition. Modus Ponendo Ponens}

Modus ponendo ponens, a mechanism for the construction of a deductive proof, is a valid rule of inference. Altogether modus ponendo ponens can be summarized as

$\mathrm{P}$ (i.e. an equation, a theorem et cetera) implies $\mathrm{Q}$ (i.e. an equation, a theorem et cetera).

$\mathrm{P}$ is (asserted or proved to be) true.

Therefore $\mathrm{Q}$ is true.

In mathematics and logic, modus ponendo ponens is a kind of a direct proof with the capacity to show the truth or falsehood of a given theorem/statement by a straightforward combination of established facts or axioms, or existing lemmas or other theorems and without making any further assumptions. In order to directly prove a conditional statement of the form "If P, then Q" it suffices to consider the situations in which the statement $\mathrm{P}$ is true. 


\subsubsection{Definition. Modus Tollendotollens}

Modus tollens or modus tollendotollens is a valid rule of inference. The modus tollens rule can be stated formally as:

If $\mathrm{P}$ (i.e. an equation, a theorem et cetera), then $\mathrm{Q}$ (i.e. an equation, a theorem et cetera).

If not $Q$, then not $P$.

Not Q.

Therefore, not $\mathrm{P}$.

More complex rewritings involving modus tollendotollens are often found in set theory.

\subsubsection{Definition. The Fallacy of Circulus in Demonstrando}

Circulus in demonstrando or circular reasoning is a logical fallacy. Circular reasoning is a type of reasoning in which the components are many times logically valid. The components of circular reasoning lead back and forth to each other, in a circle, each having only the other for support. Consequently, because the premises are true, the conclusion must be true. Circulus in demonstrando is a logical fallacy in which the proof begins with the conclusion.

\section{Example I.}

Our belief in the Bible is justified because the Bible it is the word of a God, which is existing.

Our belief in a God, which is existing is justified because it is written in the Bible.

\section{Example II.}

$\mathrm{Y}$ is true because $\mathrm{X}$ is true.

$\mathrm{X}$ is true because $\mathrm{Y}$ is true.

As a consequence the argument fails to persuade. Closely connected with the fallacy of circular reasoning (circulus in probando) is the fallacy of begging the question, a fallacy in which a prove is based on a premise which itself requires proof.

\subsubsection{Definition. The Fallacy of Petitio Principia or "Begging the Question"}

The fallacy of petitioprincipii, or "begging the question" is a type of circular reasoning which is committed when a prove is based on a premise which itself requires proof.

\subsubsection{Definition. Einstein's Mass-Energy Equivalence Relation}

Einstein's discovery of the equivalence of matter/mass and energy [14] in the year 1905 lies at the core of today's modern physics. According to Albert Einstein [15], the rest-mass om, a measure of the inertia of a (quantum mechanical) object is related to the relativistic mass ${ }_{R} m$ by the equation

$$
o_{o} m={ }_{R} m \times \sqrt[2]{1-\frac{v^{2}}{c^{2}}}
$$

Thus far and without loss of generality, the total energy of a physical system ${ }_{R} E$ is numerically equal to the product of its matter/mass ${ }_{R} m$ and the speed of light $c$ squared. We rearrange the equation above and do obtain

$$
\frac{{ }_{O} E}{{ }_{R} E}=\frac{{ }_{O} m \times c^{2}}{{ }_{R} m \times c^{2}}=\sqrt[2]{1-\frac{v^{2}}{c^{2}}}
$$

where ${ }_{O} m$ denotes the "rest" mass; ${ }_{O} E$ denotes the "rest" energy; ${ }_{R} m$ denotes the "relativistic" mass; $v$ denotes the relative velocity and $\mathrm{c}$ denotes the speed of light in vacuum.

\subsubsection{Definition. The Normalized Relativistic Energy-Momentum Relation}

Before going on to discuss the relationship between Einstein's special relativity theory and the problem of the division of zero by zero in more detail, it is only slightly more complicated to derive the general form of the normalized relativistic energy-momentum relation $[16]$ as

$$
\frac{{ }_{0} m \times{ }_{0} m}{{ }_{R} m \times{ }_{R} m}+\frac{v \times v}{c \times c}=1
$$

Under conditions of special relativity theory, there is no experimental or theoretical evidence that there are 
circumstances where the relativistic energy momentum relationship breaks down. Thus far, from the above relationship it follows that

$$
\frac{v \times v}{c \times c \times\left(1-\frac{{ }_{O} m \times{ }_{O} m}{{ }_{R} m \times{ }_{R} m}\right)}=1
$$

\subsubsection{Definition. The Time of a Stationary Observer ${ }_{R} t$ and the Time of a Co-Moving Observer ot}

Time is dependent on the observer's reference frame. Especially, clocks moving at close to the speed of light $c$ will slow down with respect to a stationary observer $R$ (observer at rest). Thus far, let ${ }_{R} t$ denote the time as measured by a stationary observer, i.e. the relativistic time. Let $o t$ denote the time as measured by a moving observer $O$. The relationship between the time ${ }_{o} t$ as measured by a clock moving at constant velocity $v$ in relation to the time ${ }_{R} t$ as measured by a clock of a stationary observer R is determined by Einstein's relativistic time dilation $[15]$ as

$$
{ }_{O} t={ }_{R} t \times \sqrt[2]{1-\frac{v^{2}}{c^{2}}}
$$

where ${ }_{O} t$ denotes time as measured by a moving observer $O ;{ }_{R} t$ denotes the time as measured by a stationary observer $R$; $v$ denotes the relative velocity between both observers and $c$ denotes the speed of light in vacuum. Equally, it is

$$
\frac{{ }_{O} t}{{ }_{R} t}=\sqrt[2]{1-\frac{v^{2}}{c^{2}}}
$$

or

$$
\frac{{ }_{O} t}{c^{2}} \times \frac{c^{2}}{{ }_{R} t}=\sqrt[2]{1-\frac{v^{2}}{c^{2}}}
$$

\section{Scholium.}

Coordinate systems can be chosen freely, deepening upon circumstances. In many coordinate systems, an event can be specified by one time coordinate and three spatial coordinates. The time as specified by the time coordinate is denoted as coordinate time. Coordinate time is distinguished from proper time. The concept of proper time, introduced by Hermann Minkowski in 1908 and denoted as ${ }_{0}$, incorporates Einstein's time dilation effect. In principle, Einstein is defining time exclusively for every place where a watch measuring this time is located.

“...Definition... der... Zeit... für den Ort, anwelchemsich die Uhr... befindet...” [15].

In general, a watch is treated as being at rest relative to the place where the same watch is located.

"Eswerdefernermittels der imruhenden System befindlichenruhenden Uhren die Zeit $t$ [R $t$, author] des ruhenden Systems... bestimmt, ebensowerde die Zeit $\tau$ [ot, author] des bewegten Systems, in welchensichrelativzuletzteremruhende Uhrenbefinden, bestimmt..." [15].

Only, the place where a watch at rest is located can move together with the watch itself. Therefore, due to Einstein, it is necessary to distinguish between clocks as such which are qualified to mark the time ${ }_{R} t$ when at rest relatively to the stationary system $R$, and the time ${ }_{o} t$ when at rest relatively to the moving system $O$.

"Wirdenkenunsfernereine der Uhren, welcherelativzumruhenden System ruhenddie Zeit $t$ [ ${ }_{R} t$, author], relativzumbewegten System ruhend die Zeit $\tau$ [ot, author] anzugebenbefähigtsind..." [15].

In English:

$<$ Further, we imagine one of the clocks which are qualified to mark the time $t\left[{ }_{R} t\right.$, author] when at rest relatively to the stationary system, and the time $\tau$ [ot, author] when at rest relatively to the moving system...>.

In other words, we have to take into account that both observers have at least one point in common, the stationary observer $R$ and the moving observer $O$ are at rest, but at rest relative to what? The stationary observer $R$ is at rest relative to a stationary co-ordinate system $\mathrm{R}$, the moving observer $O$ is at rest relative to a moving co-ordinate system $O$. Both co-ordinate systems can but must not be at rest relative to each other. The time ${ }_{R} t$ of 
the stationary system $\mathrm{R}$ is determined by clocks which are at rest relatively to that stationary system $\mathrm{R}$. Similarly, the time $o t$ of the moving system $O$ is determined by clocks which are at rest relatively to that the moving system $O$. What is the time marked by the clock when viewed from the stationary system? What is the time marked by the clock when viewed from the moving system? In last consequence, due to Einstein's theory of special relativity, a moving clock $(o t)$ will measure a smaller elapsed time between two events than that measured by a non-moving (inertial) clock $\left({ }_{R} t\right)$ between the same two events.

\subsubsection{Definition. The Normalized Relativistic Time Dilation}

As defined above, due to Einstein's special relativity, it is

$$
\frac{{ }_{O} t}{{ }_{R} t}=\sqrt[2]{1-\frac{v^{2}}{c^{2}}}
$$

The normalized relativistic time dilation relation [16] follows as

$$
\frac{{ }_{O} t^{2}}{{ }_{R} t^{2}}+\frac{v^{2}}{c^{2}}=1
$$

\subsection{Axioms}

The following theory is based on the next axiom.

\section{Axiom I. (Lex identitatis)}

$$
+1=+1
$$

(Axiom I)

\section{Results}

Albert Einstein's (1879-1955) theory of special relativity published 1905 superseded the 200-year-old theory of mechanics as created by Isaac Newton [17]. One of the features of Einstein's theory of special relativity is that all observers will measure exactly the same speed of light in a vacuum, independent of photon energy (Lorentz invariance). Meanwhile, Einstein's theory of special relativity has passed a lot of observational and experimental investigations, opportunities to test the validity of Einstein's theory of special relativity are increasing. In particular, the predictions of Einstein's theory of special relativity are still consistent with experimental data.

\subsection{Theorem. Einstein's Relativistic Energy-Momentum Relation under Conditions Where $o m=0$}

Due to Einstein's theory of special relativity the rest-mass ${ }_{\mathrm{O}} \mathrm{m}$ of a particle can be equal to zero. In this case the energy of such a particle is not destroyed but converted completely into the pure energy of a wave.

\section{Claim.}

Under conditions of special relativity (inertial frames of reference) there are circumstances, where the rest-mass (i.e. of a particle like photon) is $o m=0$. Under conditions where the rest-mass is $o m=0$ we must accept that

$$
+v \times v=+c \times c
$$

Proof.

In general, due to special relativity, it is

$$
{ }_{o} m={ }_{R} m \times \sqrt[2]{1-\frac{v^{2}}{c^{2}}}
$$

or

$$
\frac{{ }_{O} m \times{ }_{O} m}{{ }_{R} m \times{ }_{R} m}+\frac{v \times v}{c \times c}=1
$$


Under experimental conditions where ${ }_{o} m=0$ we obtain

$$
\frac{0 \times 0}{{ }_{R} m \times{ }_{R} m}+\frac{v \times v}{c \times c}=1
$$

or

$$
0+\frac{v \times v}{c \times c}=1
$$

Or

$$
+v \times v=+c \times c
$$

\section{Quod erat demonstrandum.}

\section{Scholium.}

Under experimental conditions where ${ }_{o} m=0$ Einstein's relativistic energy-momentum relation is defined. Under these circumstances the whole energy of a system does not disappear but is converted into the energy of an electromagnetic wave.

\subsection{Theorem. Einstein's Relativistic Energy-Momentum Relation under Conditions Where the Relative Velocity Is Equal to $v=0$}

Einstein's theory of special relativity is valid even under conditions where the relative velocity $v=0$. Under conditions where the relative velocity $v=0$ the wave energy (of a quantum mechanical object) as such is not destroyed but converted completely into pure energy of a "particle".

Claim.

Under conditions of special relativity (inertial frames of reference) there are circumstances, where the relative velocity $v=0$. Under conditions where the relative velocity $v=0$ we must accept that

$$
{ }_{o} m \times{ }_{O} m={ }_{R} m \times{ }_{R} m
$$

Proof.

In general, due to special relativity, it is

$$
{ }_{o} m={ }_{R} m \times \sqrt[2]{1-\frac{v^{2}}{c^{2}}}
$$

or

$$
{ }_{o} m^{2}={ }_{R} m^{2} \times\left(1-\frac{v^{2}}{c^{2}}\right)
$$

or

$$
\frac{{ }_{O} m^{2}}{{ }_{R} m^{2}}=1-\frac{v^{2}}{c^{2}}
$$

or

$$
\frac{{ }_{O} m \times{ }_{O} m}{{ }_{R} m \times{ }_{R} m}+\frac{v \times v}{c \times c}=1
$$

Under experimental conditions of special relativity where $v=0$ we obtain

$$
\frac{{ }_{O} m \times{ }_{O} m}{{ }_{R} m \times{ }_{R} m}+\frac{0 \times 0}{c \times c}=1
$$

or 


$$
\frac{{ }_{O} m \times{ }_{O} m}{{ }_{R} m \times{ }_{R} m}+0=1
$$

or

$$
\frac{{ }_{O} m \times{ }_{O} m}{{ }_{R} m \times{ }_{R} m}=1
$$

or

$$
{ }_{O} m \times{ }_{O} m={ }_{R} m \times{ }_{R} m
$$

\section{Quod erat demonstrandum.}

Scholium.

Under experimental conditions where $v=0$ Einstein's relativistic energy-momentum relation is defined.

\subsection{Theorem. The Division of Zero by Zero I}

Let us perform a thought experiment under conditions of inertial frames of reference. Experimental condition: two observers at rest relative to each other (the relative velocity $v$ is equal to zero) are moving somewhere in deep space. Thus far, let us now consider the particular case of special relativity where the relative velocity between observers is equal to $v=0$ in more detail.

Claim.

Under conditions of special relativity (inertial frames of reference) the division of zero by zero is possible and allowed. In particular, it is

$$
\frac{0}{0}=1
$$

Proof.

Due to our Axiom I it is

$$
+1=+1
$$

Multiplying this equation with $\mathrm{m}$, the "rest mass", we obtain

$$
{ }_{o} m \times 1={ }_{O} m \times 1
$$

In general, due to Einstein's special relativity it is equally

$$
{ }_{o} m={ }_{R} m \times \sqrt[2]{1-\frac{v^{2}}{c^{2}}}
$$

Thus far, the starting point of this proof by contradiction is based on the general validity of Einstein's special relativity under conditions of inertial frames of reference. Squaring Einstein's relativistic energy-momentum relation leads to

$$
{ }_{o} m^{2}={ }_{R} m^{2} \times\left(1-\frac{v^{2}}{c^{2}}\right)
$$

Collecting and rearranging together the terms one then finds straightforwardly the probability theory consistent form of the normalized relativistic energy-momentum relation [16] as

$$
\frac{{ }_{O} m \times{ }_{O} m}{{ }_{R} m \times{ }_{R} m}+\frac{v \times v}{c \times c}=1
$$

from which we find in general that

$$
+\frac{v \times v}{c \times c}=1-\frac{{ }_{O} m \times{ }_{O} m}{{ }_{R} m \times{ }_{R} m}
$$


Multiplying this equation by the speed of the light squared $c^{2}$, we obtain

$$
+v \times v=c \times c\left(1-\frac{{ }_{O} m \times{ }_{O} m}{{ }_{R} m \times{ }_{R} m}\right)
$$

The division of this equation before by the term $\left(1-\left(\left({ }_{o} m\right)^{2} /\left({ }_{R} m\right)^{2}\right)\right)$, yields the speed of the light squared $c^{2}$ as

$$
\frac{v \times v}{\left(1-\frac{{ }_{O} m \times{ }_{O} m}{{ }_{R} m \times{ }_{R} m}\right)}=c \times c
$$

Under conditions of special theory of relativity, the stationary $R$ and co-moving observer $O$ will agree on the speed of the light. In other words, the speed of the light is constant. The speed of light in vacuum, commonly denoted by c, is treated as a physical constant which is different from zero. The precise value of speed of light in vacuum is $299,792,458$ metres per second. In principle, it is possible, that the constancy of the speed of the light $\mathrm{c}$ is something relative and nothing absolute. If we follow Einstein, the speed of the light appears not to be independent of the gravitational potential, the constancy of the speed of the light $\mathrm{c}$ appears to be determined by a constant gravitational potential. Einstein:

"Dagegen bin ich der Ansicht, da $\beta$ das Prinzip der Konstanz der Lichtgeschwindigkeit sich nur insoweit aufrecht erhalten läßt, als man sich auf raum-zeitlicheGebiete von konstantem Gravitationspotential beschränkt. Hier liegt nach meiner Meinung die Grenze der Gültigkeit... des Prinzips der Konstanz der Lichtgeschwindigkeit und damit unserer heutigen Relativitätstheorie" [18].

The constancy of the speed of the light $c$ is determined by a constant gravitational potential. It is important to stress out that the speed of light in vacuum, $c$, and the gravitational constant, $G$, can be set equal to unity as in a geometrized unit system. Special relativity has many implications. We divide the equation above by the speed of the light squared $c^{2}$ and do obtain

$$
\frac{v \times v}{c \times c \times\left(1-\frac{{ }_{0} m \times{ }_{0} m}{{ }_{R} m \times{ }_{R} m}\right)}=\frac{c \times c}{c \times c}=\frac{299792458^{2}[\mathrm{~m} / \mathrm{s}]^{2}}{299792458^{2}[\mathrm{~m} / \mathrm{s}]^{2}}=1
$$

Under conditions of the theory of special relativity, this form of Einstein's relativistic energy momentum relation is generally valid. In other words, by direct substitution, under these experimental conditions $(v=0)$, we obtain

$$
\frac{0 \times 0}{c \times c \times\left(1-\frac{{ }_{O} m \times{ }_{0} m}{{ }_{R} m \times{ }_{R} m}\right)}=1
$$

Due to our theorem above (under conditions where $v=0$ ) it is equally ${ }_{O} m={ }_{R} m$ or ${ }_{O} m^{2}={ }_{R} m^{2}$. Thus far, we are substituting $o m^{2}$ by ${ }_{R} m^{2}$ and do obtain

$$
\frac{0 \times 0}{c \times c \times\left(1-\frac{{ }_{R} m \times{ }_{R} m}{{ }_{R} m \times{ }_{R} m}\right)}=1
$$

or

$$
\frac{0 \times 0}{c \times c \times(1-1)}=1
$$

or

$$
\frac{0 \times 0}{c \times c \times 0}=1
$$


Under conditions of the special theory of relativity it follows that

$$
\frac{0}{0}=1
$$

\section{Quod erat demonstrandum.}

Experimental mathematics is one of the many approaches to mathematics. As in experimental science, experimental mathematics can be used to investigate mathematical objects, to identify properties and patterns and to provide us with fundamental insights through the use of (properly constructed "thought") experiments.

\subsection{Theorem. The Normalization of the Relationship between the Time as Measured by a Moving Observer $\boldsymbol{O}$ and the Time as Measured by a Observer at Rest $\boldsymbol{R}$}

Let ${ }_{R} t$ denote the time as measured by a stationary observer and let ${ }_{o} t$ denote the time as measured by a moving observer. The relationship between the time as measured by a stationary observer and the time as measured by a moving observer can be normalized and generalized as

$$
\frac{{ }_{R} t-{ }_{0} t}{{ }_{R} t-{ }_{0} t}=+1
$$

\section{Direct proof.}

Due to our Axiom I it is

$$
+1=+1
$$

Multiplying this equation with ${ }_{R} t$ the time as measured by a stationary observer, we obtain

$$
1 \times{ }_{R} t={ }_{R} t \times 1
$$

We subtract the time ${ }_{o} t$ as measured by a moving observer from the equation above. It is

$$
{ }_{R} t-{ }_{o} t={ }_{R} t-{ }_{o} t
$$

Now, we divide the term $\left({ }_{R} t-o t\right)$ by the term $\left({ }_{R} t-o t\right)$ itself and do obtain

$$
\frac{{ }_{R} t-{ }_{0} t}{{ }_{R} t-{ }_{0} t}=+1
$$

\section{Quod erat demonstrandum.}

\section{Scholium.}

The prove above is not based on a premise or on an axiom which itself requires a proof since $+1=+1$ is true. Consequently, the fallacy of petitioprincipii or "begging the question" is not committed. The fallacy of circulus in demonstrando is not committed since the proof does not begin with the conclusion $\left({ }_{R} t-{ }_{o} t\right) /\left({ }_{R} t-{ }_{o} t\right)=1$. The proof begins with the axiom that $+1=+1$, which is correct. Form this axiom the conclusion drawn is that ${ }_{R} t-$ $\left.{ }_{o} t\right) /\left({ }_{R} t-{ }_{o} t\right)=1$. The above formula can be proofed by physical experiments. The above theorem justifies a transition from an axiom to an experiment and thus far to the testable consequences. Equally significant is the capacity of the above theoretical structure which leads to consequences that can be compared with experience.

\subsection{Theorem. The Division of Zero by Zero II}

Let ${ }_{R} t$ denote the time as measured by a stationary observer and let ${ }_{o} t$ denote the time as measured by a moving observer. If Einstein's special relativity is correct and valid, then under conditions where ${ }_{R} t={ }_{O} t$ it is

$$
\frac{{ }_{R} t-{ }_{O} t}{{ }_{R} t-{ }_{O} t}=\frac{+0}{+0}=+1
$$

Proof by modus ponendo ponens.

Due to our Axiom I it is

$$
+1=+1
$$


Due to the theorem before this is equivalent to

$$
\frac{{ }_{R} t-{ }_{O} t}{{ }_{R} t-{ }_{O} t}=+1
$$

Einstein's special relativity covers even the case if ${ }_{R} t={ }_{o} t$. In this case, the relative velocity between the stationary observer and the moving observer is $v=0$. Thus far, it is ${ }_{R} t-{ }_{o} t=0$. We obtain

$$
\frac{{ }_{R} t-{ }_{O} t}{{ }_{R} t-{ }_{O} t}=\frac{+0}{+0}=+1
$$

Consequently due to the requirements of a proof by modus ponendo pones if $P$ then $Q$, we obtain the following premise: if Einstein's special relativity is valid, then

$$
\frac{+0}{+0}=+1
$$

Einstein's special theory of relativity predicts a lot of phenomena that seem weird. But special theory of relativity has passed a huge number of experimental tests. The experimental observations are still consistent with the predictions of special relativity. The results of all known experiments are that Einstein's special theory of relativity is correct and valid. Consequently, $P$ (or Einstein's special relativity) is (asserted or proved to be) true. Therefore $Q($ i.e. $0 / 0=1)$ is true or

$$
\frac{+0}{+0}=+1
$$

\section{Quod erat demonstrandum.}

\section{Scholium.}

A number of experiments can be performed to proof the above relationship. It is known that clocks on orbiting satellites move slower by a certain amount. Further, atomic clocks on planes move slower too, compared to identical stationary clocks on earth. An atomic clock on the moon will run slower compared to an identical atomic clock on the earth and can be used to test the structure above.

\section{Discussion}

In general, mathematical expressions which are not definitively or precisely determined are said to be indeterminate. The term indeterminate forms was originally introduced by François-Napoléon-Marie Moigno (18041884), a student of Cauchy, in the middle of the 19th century. In principle, several types of indeterminate forms are distinguished. Some of the indeterminate forms typically considered in the literature are denoted by $0 / 0$ or by $\infty / \infty$ and equally by $0 \times \infty$ or by $\infty-\infty$ or by $0^{\circ}$ or by $1^{\infty}$ and by $\infty^{\circ}$.

Thus far, for thousands of years, at least since Aristotle, the division of zero by zero was not allowed. With that brief sketch of the historical background of indeterminate forms, we resolved this vagueness by using Einstein's special theory of relativity. Einstein's special theory of relativity determines very precisely what happens, if 0 is divided by 0 . Following Einstein's special theory of relativity, the division of 0 by 0 is not indeterminate at all, the division of 0 by 0 is determinate as $(0 / 0)=1$. As is known, Einstein's theory of special relativity has passed a lot of tests, the experiments supporting the validity of Einstein's theory of special relativity are increasing. In particular, the predictions of Einstein's theory of special relativity are still and without any contradiction consistent with experimental data. In this paper, we have used Einstein's theory of special relativity to demonstrate that the division of zero by zero make sense and is defined without any contradictions. The thought experiments are properly constructed. Especially Equation (37) is just a reformulation of Einstein's relativistic energy momentum relation and is as such defined at any event under conditions of special theory of relativity. Following the predictions of Einstein's theory of special relativity, we must accept that $(0 / 0)=1$.

There are, however, differences in the way how to treat the division of 0 by 0 . Contrary to Einstein's special theory of relativity, L'Hospital's Rule is undoubtedly founded on the assumption that it is just not clear what is happening in the limit. Thus far, L'Hospital's Rule, named after the $17^{\text {th }}$ century French mathematician Guillaume de L'Hôpital and published in his 1696 book Analyse des Infiniment Petits pour l'Intelligence des Lignes 
Courbes claims to tell us all we need and all we have to do if we have an indeterminate form. As already pointed out the general form of L'Hôpital's rule may cover many cases. In the light of this publication, it appears to be possible that L'Hôpital's rule is not generally valid. The general validity of L'Hôpital's rule should be reviewed from the beginning.

As it is, we are always and already linked to the historical development of science as such. Altogether, the division of zero by zero is possible, allowed and defined. But the division of zero by zero can lead to some paradoxes if some specific rules of precedence on which the division of zero by zero is grounded, are not respected.

\section{Example I.}

Clearly, it is incorrect that $1=2$. Multiplying this equation by 0 it is $1 \times 0=2 \times 0$ and we obtain $0=0$ which is correct. Dividing by zero, it is $(0 / 0)=(0 / 0)$ and due to our finding $(0 / 0)=1$ we obtain $1=1$ which is of course correct. Thus far, we started with something incorrect by claiming that $1=2$. After the division of zero by zero we obtained $1=1$, i.e. something correct. This is a contradiction. Under these circumstances, we may infer that we are not allowed to divide by zero since we obtained an erroneous result. Contrary to facts, the erroneous result obtained is due to the problem of the multiplication by zero. Consequently, a multiplication by 0 can lead to an erroneous result since something obviously false $($ i.e. $1=2)$ is converted into something true $($ i.e. $0=0)$. In other words, from something incorrect follows something correct. The multiplication by zero is much more problematic than the division by zero. Altogether, a division by zero appears to possess a greater priority then the multiplication by zero. Respecting this fact we get another picture.

\section{Example II.}

Again, it is incorrect that $1=2$. Multiplying this equation by 0 it is $1 \times 0=2 \times 0$. Dividing by zero, it is $((1 \times$ $0) / 0)=((2 \times 0) / 0)$. Under circumstances where the division by zero is performed prior to the multiplication by zero we obtain $(1 \times(0 / 0))=(2 \times(0 / 0))$. Since $0 / 0=1$, it follows that $1=2$ which is equal to the (incorrect) starting point of this example. In other words, as mentioned previously, the division of zero by zero is possible, allowed and defined. But to avoid some paradoxes while performing the division of zero by zero some specific rules of precedence on which the division of zero by zero is grounded, should be worked out in detail and respected. The multiplication by zero appears to be not less difficult then the division by zero.

\section{Example III.}

Once again, it is incorrect that $1=2$. Multiplying this equation by 0 it is $1 \times 0=2 \times 0$. Dividing by zero, it is $((1 \times 0) / 0)=((2 \times 0) / 0)$. We rearrange this equation and do obtain $((1 / 0) \times 0)=(2 \times(1 / 0) \times 0)$. Following Wallis, who claimed in 1656 that " $1 / \infty \ldots$ habendaerit pro nihilo" [19] we obtain another picture. Thus far, under circumstances where $(1 / 0=\infty$ and $1=0 \times \infty$ and $0 / 0=1)$ we obtain $((\infty) \times 0)=(2 \times(\infty) \times 0)$ which is equivalent to our (incorrect) starting point $1=2$.

In particular, opponents of this approach to the problem of the division of 0 by 0 may attempt to discredit this contribution in both personal and professional ways. A common technique used by opposing authors is to create the impression that a proof is based on a logical fallacy or the result of a proof is grounded on a logical fallacy. The speed of the light (Theorem 3.3, Equation (36)) is determined very precisely. Dividing this equation by the speed of the light $c^{2}$, we obtain

$$
\frac{v \times v}{c \times c \times\left(1-\frac{{ }_{O} m \times{ }_{O} m}{{ }_{R} m \times{ }_{R} m}\right)}=\frac{c \times c}{c \times c}=\frac{299792458^{2}[\mathrm{~m} / \mathrm{s}]^{2}}{299792458^{2}[\mathrm{~m} / \mathrm{s}]^{2}}=1
$$

Under conditions of special relativity, there are circumstances where $v=0$. In this case, it follows that $0 / 0=1$. Thus far, the theorem 3.5 is not based on the division of zero by zero too. In theorem 3.5 a term $\left({ }_{R} t-{ }_{o} t\right)$ is divided by itself, i.e. by $\left({ }_{R} t-{ }_{o} t\right)$. Due to the rules of mathematics and based on the achievements of special theory of relativity the result is

$$
\frac{{ }_{R} t-{ }_{O} t}{{ }_{R} t-{ }_{O} t}=+1
$$

nothing more but nothing less too. In accordance with special theory of relativity, the term $\left.{ }_{R} t-{ }_{o} t\right)$ can take many different values. Since it is possible and allowed in real life that the time ${ }_{R} t$ as measured by a stationary observer $R$ is identical with the time $o t$ as measured by a co-moving observer $O$, it is natural, possible and al- 
lowed that ${ }_{R} t={ }_{o} t$ and thus far that $\left({ }_{R} t-{ }_{o} t\right)=0$. This is a real-life situation. In this case and of course under conditions where Einstein special theory of relativity is still valid, we obtain $\left({ }_{R} t-{ }_{o} t\right) /\left({ }_{R} t-{ }_{o} t\right)=0 / 0=1$. Real life experiments or practice as such and not a highly abstract theoretical framework difficult to understand and much more difficult to proof tell us exactly what happens, if we are faced with situations, where zero is divided by zero.

\section{Conclusion}

The general problem of the division of zero by zero is solved. In general, under conditions of special relativity, it is $(0 / 0)=1$. Thus far, while the problem of the division of zero by zero is solved, new problems are created too. It appears to be necessary to review the general validity of L'Hôpital's rule and to work out the rules of precedence in detail, when performing some algebraic operations with zero.

\section{References}

[1] Bibhutibuhusan, D. (1927) Early History of the Arithmetic of Zero and Infinity in India. Bulletin of the Calcutta Mathematical Society, 18, 165-176.

[2] Aristotle (1930) Physica. Vol. 2. In: Hardie, R.P. and Gaye, R.K., Eds., The Works of Aristotle, Translated by Ross, W.D., Calenderon Press, Oxford, Physica Book 4, 8, 215 b, Line 13.

[3] Boyer, C.B. (1943) An Early Reference to Division by Zero. The American Mathematical Monthly, 50, 487-491. http://dx.doi.org/10.2307/2304187

[4] Nicomachus of Gerasa (1926) Introduction to Arithmetic. Translated by D’Ooge, M.L. New York, pp. 48, 120, 237238.

[5] Colebrooke, H.T. (1817) Algebra, with Arithmetic and Mensuration, from the Sanscrit of Brahmagupta and Bhaskara. John Murray, London, 339-340.

[6] Colebrooke, H.T. (1817) Algebra, with Arithmetic and Mensuration, from the Sanscrit of Brahmagupta and Bhaskara. John Murray, London, 137-138.

[7] Wallisii, J. (1655) De sectionibusconicis, nova methodoexpositis, tractatus. Typis Leon: Lichfield Academix Typographi, Impensis Tho. Robinson, Oxonii, p. 4.

[8] Wallisii, J. (1656) Arithmeticainfinitorum, Sive Nova methodusinquirendi in curvilineorumquadraturam, aliaqdifficilioraproblematamatheseos. Typis Leon: LichfieldAcademixTypographi, ImpensisTho. Robinson, Oxonii, p. 152.

[9] Romig, H.G. (1924) Discussions: Early History of Division by Zero. The American Mathematical Monthly, 31, 387389. http://dx.doi.org/10.2307/2298825

[10] Newton, I. (1744) Opuscula Mathematica Philosophica et Philologica. In Tres Tomos Distributa. Tomus Primus. Joh. Castillionues, Juris consultus, Lusannaeet Genevae, 4. http://dx.doi.org/10.3931/e-rara-8608

[11] Berkeley, G. (1754 [MDCCLIV]) The Analyst: Or, A Discourse Addressed to an Infidel Mathematician. Wherein It Is Examined Whether the Object, Principles, and Inferences of the Modern Analysis Are More Distinctly Conceived, or More Evidently Deduced, than Religious Mysteries and Points of Faith. 2nd Edition, Printed for J. and R. Tonson and S. Draper in the Strand, London, 59.

[12] Bergstra, J.A., Hirshfeld, Y. and Tucker, J.V. (2009) Meadows and the Equational Specification of Division. Theoretical Computer Science, 410, 1261-1271. http://dx.doi.org/10.1016/j.tcs.2008.12.015

[13] Michiwaki, H., Saitoh, S. and Yamada, M. (2016) Reality of the Division by Zero z/0=0. International Journal of Applied Physics and Mathematics, 6, 1-8. http://dx.doi.org/10.17706/ijapm.2016.6.1.1-8

[14] Einstein, A. (1905) Ist die Trägheit Eines Körpers von Seinem Energieinhalt Abhängig? Annalen der Physik, 323, 639641.

[15] Einstein, A. (1905) Zur Elektrodynamik Bewegter Körper. Annalen der Physik, 322, 891-921.

[16] Barukčić, I. (2013) The Relativistic Wave Equation. International Journal of Applied Physics and Mathematics, 3 , 387-391.

[17] Newton, I. (1686) Philosophiae Naturalis Principia Mathematica. S. Pepys, London.

[18] Einstein, A. (1912) Relativität und Gravitation. Erwiderung auf Eine Bemerkung von M. Abraham. Annalen der Physik, 343, 1059-1064. http://dx.doi.org/10.1002/andp.19123431014

[19] Wallisii, J. (1656) Arithmetica Infinitorum, Sive Nova Methodus in Quirendi in Curvilineorum Quadraturam, Aliaq Difficiliora Problemata Matheseos. Typis Leon: Lichfield Academix Typographi, Impensis Tho. Robinson, Oxonii, 152. 